Background and aims Liquid formulations are easy to swallow but they may have disadvantages such as a bad taste, preservatives or restricted storage conditions. These disadvantages may be overcome by oral solid flexible dosage forms such as powders or minitablets. The aim of this study was to investigate the acceptance and preference of four oral dosage forms in children aged 1-4 years in the Netherlands.

Methods Parents administered four different placebo formulations: a 4-mm round, uncoated mini-tablet, powder, suspension and syrup at home to their (healthy) child twice on one day following a randomized cross-over design. They were asked to report the child acceptance by the result of the intake and by a child acceptance score on a $10-\mathrm{cm}$ visual analogue scale (VAS). At the end of the study parents were asked to report the child and parent preference. Results 183 children were included and 151 children were evaluated. The mini-tablet was fully swallowed in $97 \%$ of all cases, the powder $81 \%$, the suspension $86 \%$ and the syrup $83 \%$. The mean VAS-scores were: tablet 9.01; powder 8.00; suspension 7.78; syrup 8.04. The mini-tablet was significantly better accepted than the three other dosage forms $(\mathrm{p}<0.05)$. The same pattern was observed when only the first intake was considered given a carry-over effect. Children and parents preferred the tablet and syrup over the suspension and the suspension over the powder $(\mathrm{p}<0.05)$.

Conclusions All dosage forms were well accepted, but the minitablets were the best accepted and preferred dosage form.

\section{SOCIOECONOMIC STATUS IN RELATION TO LIPID AND GLUCOSE METABOLISM IN EARLY CHILDHOOD. THE ABCD-STUDY}

doi:10.1136/archdischild-2012-302724.0218

${ }^{1,2} \mathrm{G}$ van den Berg, ${ }^{2} \mathrm{M}$ van Eijsden, ${ }^{3} \mathrm{TGM}$ Vrijkotte, ${ }^{1} \mathrm{RJBJ}$ Gemke. ${ }^{1}$ Pediatrics, VU University Medical Center; ${ }^{2}$ Epidemiology, Documentation and Health Promotion, Public Health Service of Amsterdam; ${ }^{3}$ Public Health, Academic Medical Center, University of Amsterdam, Amsterdam, The Netherlands

Objective The objective of this study was to explore the relations of socioeconomic status to lipid and glucose metabolism as indicators of cardiovascular health in 5-6 year olds.

Methods In 1308 5-6 year old ethnic Dutch children from the ABCD cohort study, lipids (cholesterol, LDL-C, HDL-C, triglycerides), glucose and C-peptide ( $\mathrm{n}=974$ ) were measured after an overnight-fast. Insulin resistance was calculated with HOMA. Using linear regression the association of lipid and glucose metabolism to socioeconomic status as indicated by maternal education and income adequacy was examined.

Results There were no differences in cholesterol, HDL-C, LDL-C, and triglycerides between socioeconomic groups. However, children with low educated mothers had on average a higher glucose $(p=0.01)$, C-peptide $(p=0.001)$, and insulin resistance $(p=0.001)$ compared to children with high educated mothers. These associations could not be explained by birth weight, maternal BMI, breastfeeding duration, and physical activity. Childhood BMI partly explains these associations, but after adjustment for BMI the association between maternal education and markers of the glucose metabolism remained significant (models controlled for age, height, and sex).

Conclusion Socioeconomic status appears to be an independent risk factor for cardiovascular function and seems to emerge in early childhood. In absence of underlying mechanisms these empirical findings are relevant for public health care and further explanatory research.

\section{INCIDENCE TESTING OF HUNTER SYNDROME IN A POPULATION AT RISK - FIRST RESULTS OF A BINATIONAL SCREENING PROGRAMME}

doi:10.1136/archdischild-2012-302724.0219
1J Gödeke, ${ }^{1} \mathrm{C}$ Güth, ${ }^{2} \mathrm{C}$ Lampe, ${ }^{2} \mathrm{~J}$ Reinke, ${ }^{3} \mathrm{~F}$ Lagler, ${ }^{4} \mathrm{Z}$ Lukacs, ${ }^{2} \mathrm{M}$ Beck, ${ }^{1} \mathrm{D}$ von Schweinitz. 'Department of Pediatric Surgery, Dr. von Haunersches Children's Hospital, LMU, Munich; 'Villa Metabolica, Children's Medical Center, Johannes Gutenberg University of Mainz, Mainz, Germany; ${ }^{3}$ nstitute for Congenital Metabolic Diseases, Paracelsus Medical University of Salzburg, Salzburg, Austria; ${ }^{4}$ Children's Hospital, University Medical Center Hamburg - Eppendorf, University of Hamburg, Hamburg, Germany

Background Hunter syndrome (Mucopolysaccharidosis type II; $\mathrm{X}$-linked inheritance; prevalence rate in Europe approximately 1:77000 male newborns) is a rare, progressive, multisystemic disease, caused by deficiency of the lysosomal enzyme Iduronate-2sulfatase. Due to the very heterogeneous phenotype Hunter syndrome is often not diagnosed before pre-school age. This is unfortunate, because patients would significantly benefit from the earliest possible start of treatment containing enzyme replacement therapy.

Early screening methods are possible, but due to the rarity of this disease they are too expensive to be performed in all newborns. An at-risk patient population screening provides opportunity for timely identification of the patients. All children with Hunter syndrome have an umbilical hernia and about $60 \%$ develop an inguinal hernia in early childhood. This is significantly more than in the general population

Methods Since February 2012 an at-risk population screening (male sex $<18$ years old, presence of an umbilical hernia, surgery for inguinal hernia) is carried out in Germany and Austria. Test centers are over 90 surgical test centers which operate on children routinely. Patients are screened via Iduronate-2-sulfatase enzymatic assay from dried blood spots cards and an additional questionnaire.

Results and conclusion We will present the first results after 6 months study period. In case of positive screening results, a routine screening for the above mentioned at-risk population should be discussed. Simultaneously, an extended study within the European Union can then be planned and organized.

Acknowledgements This work is supported by a research-grant from Shire ${ }^{\circledR}$ Deutschland GmbH.

\section{CORD BLOOD CHEMERIN AND OBESTATIN IN LARGE FOR GESTATIONAL AGE INFANTS}

doi:10.1136/archdischild-2012-302724.0220

T Boutsikou, DD Briana, A Dendrinou, M Boutsikou, L Stamati, S Baka, D Hassiakos, D Gourgiotis, A Malamitsi-Puchner. Athens University Medical School, Athens, Greece

Background and aims Increased neonatal adiposity is associated with childhood obesity and subsequent development of adult diseases. Chemerin was recently introduced as a novel adipocytokine inducing insulin resistance and regulating maternal-fetal metabolic homeostasis during pregnancy. Obestatin is a peptide hormone involved in the control of insulin secretion and adipocyte function. This study aimed to assess circulating concentrations of chemerin and obestatin in fetal samples from large-for-gestational-age-(LGA) and appropriate-for-gestational-age-(AGA) pregnancies and investigate their association with gender, parity and delivery mode.

Methods Cord blood chemerin and obestatin concentrations were prospectively measured by enzyme-linked immunosorbent assay in 40 LGA ( 9 born from diabetic mothers and 31 born from nondiabetic mothers) and 40 AGA singleton full-term infants.

Results Cord blood chemerin concentrations were significantly higher in LGA compared to AGA neonates, after controlling for confounding factors $(b=38.91, p<0.001, S E 9.29)$. In contrast, no significant differences in obestatin concentrations were observed between groups. Cord blood concentrations of both hormones did not depend on gender, parity or delivery mode.

Conclusions Higher chemerin concentrations in LGA neonates, possibly implying predisposition to insulin resistance, may serve as 